\title{
El edificio de altura. Una revisión de las aproximaciones sociológicas para su estudio
}

\author{
The high-rise building. A review of sociological approaches for study
}

Jorge Vergara Vidal*

Fecha de recepción:12 de agosto de 2017

Fecha de aceptación: 01 de noviembre de 2017

\section{Resumen}

La verticalización de las ciudades chilenas es un fenómeno que concita el interés público, aunque requiere de una comprensión que excede las razones económicas y las posibilidades técnicas que llevan a la proliferación de edificios de altura. El presente trabajo revisa en la literatura sociológica el tratamiento que se ha dado a los edificios de altura, con el objetivo de encontrar ciertas claves que colaboren con el estudio actual de estas formas arquitectónicas. Las conclusiones identifican un giro epistemológico que ha permitido contar con herramientas capaces de enlazar, por una parte, los aspectos arquitectónicos, materiales y técnicos implicados en los edificios de altura, junto con el análisis sociológico de los mismo.

Palabras clave: edificios de altura, objetos sociotécnicos, sociología de la arquitectura, sociología urbana, verticalización.

\begin{abstract}
The verticalization of Chilean cities is a phenomenon that attracts the public interest and requires an understanding that exceeds the economic reasons and technical possibilities that lead to the proliferation of high buildings within them. This paper reviews in the sociological literature the treatment given to high-rise buildings with the aim of providing clues that collaborate with the social study of these architectural forms. The conclusions identify an epistemological turn that has allowed tools to link the architectural, material and technical aspects involved in high-rise buildings with the sociological analysis of these.
\end{abstract}

Keywords: high-rise buildings, sociotechnical objects, sociology of architecture, urban sociology, verticalization

* Filiación: Departamento de Sociología, Facultad de Ciencias Sociales, Universidad de Chile, Santiago, Chile. Correo: jvergaravidal@uchile.cl

Este artículo se inserta en el marco del proyecto Fondecyt N³170016

Cómo citar: Vergara Vidal, J. (2017). El edificio de altura. Una revisión de las aproximaciones sociológicas para su estudio. Revista de Urbanismo, 37, 1-19. https://doi.org/10.5354/0717-5051.2017.47059 


\section{Introducción}

\section{Alturas artificiales}

Las alturas artificiales no son recientes en la historia, ni esperaron a la constitución de las grandes ciudades para aparecer, aunque claramente se consolidaron con ellas. Su práctica está manifiestamente presente en los antiguos "tells", elaborados como base de los asentamientos humanos de oriente cercano y que dieron origen a los primeros poblados (Delfante, 2006). Esa técnica, que "levantaba" las agrupaciones de edificaciones sobre un montículo de tierra y piedras dándoles altura y perspectiva, facilitando la gestión de la vida cotidiana y la defensa a partir de una separación de planos, constituye una de las bases del desarrollo de las ciudades.

El hecho de producir la diferenciación de planos mediante la producción artificial de una altura permitió vincular la posibilidad técnica (tékhné) con la utilidad política y organizar, a lo menos, dos regímenes de gestión espacial sobre la base de la ruptura y rearticulación de lo plano. El resultado de esto no generó más espacio, pero sí lo reconfiguró en un régimen simple (arriba/abajo) que ordenó las posiciones de los individuos, de las cosas y del mismo espacio ya fragmentado en diversas especies (Crang \& Thrift, 2000).

El efecto relacional de la técnica de la altura fue la escala, una noción instrumental de equilibrio a partir de la proporcionalidad de espacios y volúmenes dentro de un plano (Adler, Brittain-Catlin \& Fontana-Giusti, 2012; Herod, 2011). La escala es el fundamento del ordenamiento urbano y del diseño de su planta, pero en tanto "sentido del equilibrio" representa la base de cualquier composición espacial o de cualquier especie de espacio (Crang \& Thrift, 2000; Lussault, 2015).

Al surgir y proliferar los modernos edificios de altura la escala (el equilibrio proporcional) de las ciudades y edificaciones se vieron alteradas en varios sentidos. EI arquitecto Louis Henry Sullivan, en su The tall office building artistically considered (El alto edificio de oficinas artísticamente considerado, 1896), dio cuenta del cambio que esto implicaba respecto de la forma y la estética de los ambientes urbanos, pero a su vez señaló que estas inéditas formas arquitectónicas también buscaban un equilibrio, no en relación con la composición urbana, sino con la distribución del espacio en los centros de las ciudades, cuya escasez y elevados precios llevaban a producirlo verticalmente. Para Sullivan, los edificios de altura no son un capricho cumplido por la presencia de las condiciones técnicas para realizarlos, sino una expresión técnica de las necesidades e intereses de una sociedad.

Por su parte, el ingeniero Himmelwright (1896), junto con elogiar el logro técnico de las altas construcciones, su importancia comercial y la gran cantidad de personas que desarrollaban sus labores en ellas, hacía hincapié en la debilidad de las normativas que gobernaban los nuevos edificios, las que no solo permitían una aglomeración peligrosa de estos en las manzanas centrales, sino que tampoco regulaban adecuadamente la calidad de los materiales con los que eran construidos o con los que eran instalados sus ascensores. Su cercanía, su flamabilidad y la baja calidad de sus aceros no eran opciones de ingeniería: Himmelwright las indica como vulnerabilidades motivadas por el ahorro de costos en la construcción y la maximización de ganancias, para cuya corrección se requería un régimen urbano consciente de la relación entre lo técnico y lo social.

Para 1896, año en el que escriben Sullivan e Himmelwright, aunque llamaran la atención las alturas artificiales, estas no habían sido predominantes en la arquitectura de las ciudades y tampoco las ciudades habían sido predominantes dentro de la habitabilidad humana (Glaeser, 2011). Pero eso comenzó a cambiar y, en la actualidad, tanto la urbanización como los edificios de altura se incrementan con intensidad, constituyéndose en uno de los registros sociotécnicos más característicos de las ciudades (Grubbauer, 2010; Kärrholm, 2013; King, 2010).

Actualmente, el edificio de altura ya no es solo un "alto edificio de oficinas". En ciudades como el Santiago Metropolitano su uso residencial se ha hecho mayor que su uso comercial, y sus formas han copado las cuadras de las comunas centrales y pericentrales (Vergara Vidal, 2017). El poblamiento intensivo de estas zonas urbanas, el alto valor del suelo y las costosas implicancias ecoenergéticas de la expansión horizontal de las ciudades han puesto a los edificios de altura en el papel de actores y agencias alternativos al modelo de la ciudad expandida, apoyada principalmente en la forma antinómica de las casas (Contreras, 2011; 2017; Hidalgo y Janoschka, 2014).

Tal como lo sostenían Sullivan e Himmelwright, los edificios de altura convocan la movilización de las 
capacidades sociotécnicas de las sociedades actuales, es decir, conectan y enfrentan intereses, movilizan recursos humanos y no humanos, generan parámetros normativos para las interacciones, promueven materialidades $y$ desincentivan otras, levantan y hunden industrias, se replican como actos sociotécnicos: constituyen un particular régimen de prácticas en torno a la producción y habilitación del espacio en la altura, pero no siempre colaboran con la producción de una coherencia urbana, como lo evidencian las constantes controversias en torno a la relación edificación en altura y ciudad ${ }^{1}$.

En el campo de la arquitectura, los estudios respecto de edificios en altura, sus autores y técnicas, no son escasos, como tampoco lo son los trabajos que actualmente utilizan los edificios de altura como una variable significativa para el análisis y planificación de las ciudades (Gehl, 2011; Mohsenin \& Sevtsuk, 2013; Salasses, Schechtner \& Hidalgo, 2013; Sevtsuk \& Kalvo, 2017), pero el mayor protagonismo y proliferación que adquieren los edificios en altura como soporte para la densificación de las ciudades -y que da pie al fenómeno de la verticalización urbana-, ha abierto también la necesidad de su observación por parte de las ciencias sociales.

En el texto que sigue se desarrolla una revisión de las formas en las que la sociología ha abordado el fenómeno de las edificaciones urbanas $y$, en especial, de los edificios en altura. Su objetivo fue identificar los aspectos y enfoques que podrían ser considerados a la hora de abordar el estudio sociológico de la arquitectura y de las edificaciones de altura, ante la impresión de que las diversas aproximaciones sociológicas a los fenómenos urbanos habían puesto escaso interés en su estudio específico.

La metodología utilizada implicó una revisión exhaustiva de los autores centrales de la sociología, en busca de referencias específicas a las edificaciones consideradas como objetos de estudio sociológico. Los resultados de este proceso se detallan a continuación en un relato que, por defecto, tiende a parecer guiado por la

\footnotetext{
1 Algunos ejemplos disponibles en http://www.emol.com/noticias/Nacional/2017/04/11/853675/Guetosverticales-Intendente-Metropolitano-pide-a-inmobiliarias-que-seautorregulen-a-la-hora-de-construir.html, así como también en http://www.emol.com/noticias/Economia/2017/04/11/853699/Dirigen te-de-la-CChC-y-guetos-verticales-Hay-una-debil-regulacion-y-una-altademanda.htm
}

cronología pero que, en realidad, está orientado por los cambios operados en la epistemología y los objetos de estudio de la sociología.

El texto se organiza en torno a tres momentos disciplinares que consideran: la etapa de desarrollo temprano de la sociología; la de desarrollo, consolidación y crisis de la sociología urbana como subcampo disciplinar; y la etapa de emergencia y consolidación del espacio como objeto de estudio, que marca un giro en las investigaciones relacionadas con las ciudades y las edificaciones.

Las conclusiones de este ejercicio indican que solo en este último giro, y en virtud de las cualidades del espacio como objeto de estudio, la sociología ha podido contar con las herramientas epistemológicas capaces de enlazar los fenómenos arquitectónicos, técnicos y materiales implicados en los edificios de altura con el estudio de las asociaciones, que le son propios. Esto ha permitido tres tipos de desarrollos diferentes, dependiendo de que el espacio (edificación) sea considerado como un bien, un territorio o una técnica, y/o que sea operacionalizado conceptualmente como un "lugar" o como un "objeto".

\section{El edificio de altura en la sociología temprana}

Resulta difícil encontrar casos de observación o menciones específicas a edificaciones y/o a obras de arquitectura dentro del cuerpo de textos y autores que pueden ser considerados como parte de la etapa de desarrollo temprano de la sociología, esto es considerando desde los primeros trazados disciplinares realizados por Saint Simon, Comte, Le Play y Quetelet (1820 aproximadamente), hasta su consolidación como cuerpo específico de conocimiento mediante las cátedras y las primeras escuelas universitarias (cerca de 1920) (Lamo de Espinosa, 2001).

Durante esta etapa, marcada por el crecimiento poblacional de las ciudades europeas y la base urbana de los "estados nación", los esfuerzos de los practicantes de la disciplina estuvieron centrados en la delimitación de su objeto de estudio y la discusión en torno a los métodos e instrumentos por utilizar de modo que, aunque a su alrededor cambiaban las formas arquitectónicas y urbanas, los intereses disciplinares se enfocaron en las interacciones que se daban dentro de ellas, más que de los aspectos de la materia y la forma. 
Con todo, era imposible no tomar en cuenta la influencia de las ciudades en las acciones de los individuos, sobre todo por su vínculo con la organización de los estados modernos, y la sociología temprana, si bien casi no menciona en específico a las edificaciones y sus formas, abordó desde varios puntos de vista el fenómeno de la urbanización.

En Alemania, el efecto del cambio urbano en la reconfiguración de los tipos de vínculos entre las personas fue tempranamente considerado por Ferdinand Tönnies al distinguir, en 1887, entre vínculos esenciales (sanguíneos, comunitarios) y arbitrarios (electivos, sociales) que se enlazaban en diversos planos de análisis (Tönnies, 1947). Sus conclusiones fueron acompañadas por los intentos de Georg Simmel (2009; 2014) de articular una explicación estable respecto de las formas espaciales que asumía la interacción entre individuos y de su interés por observar los efectos de las ciudades en sus habitantes y sus tipologías.

Por su parte, los tipos de ciudades considerados a partir de su historia y su función económico-productiva predominante fueron observados tanto por Werner Sombart (1958; 1988) como por Max Weber (2005), mientras que los mercados asociados al suelo urbano fueron analizados tanto por Friedrich Engels (1965; 1976), considerando el problema del valor de las viviendas, como por Alfred Weber (Friedrich, 1929), considerando el problema de la localización de las industrias y el valor del suelo. Como es posible ver, en ninguno de estos trabajos se aborda en específico la ciudad como fenómeno material y/o técnico y menos aún las formas arquitectónicas dentro de ellas.

Max Weber tuvo, entre los nombrados, la posibilidad de visitar Nueva York y Chicago en 1904 en el periodo en que sus centros urbanos ya estaban poblados de altas edificaciones. De su experiencia, Marianne Weber declara su sorpresa con las "poderosas bestias de más de treinta pisos que se alzan frente a nosotros" en referencia a los edificios Park Row y St. Paul, a los cuales Max llama "fortalezas del capitalismo" (Scaff, 2011, p. 27), pero fuera de algunos comentarios entusiasmados a la racionalidad expresada en tales construcciones, Weber no dedicó mayores observaciones a estas formas arquitectónicas.

Dentro de la sociología francesa, por otro lado, Emile Durkheim (1975) abordó solo tangencialmente el problema de la morfología urbana puntualmente en la reseña a un libro de Henrí Francotte referido a la formación de las ciudades griegas escrito en 1903. Durkheim llama la atención sobre el sinecismo (la cohabitación de grupos elementales diversos en el Ática), indicando que este fenómeno no es solo político, sino también morfológico y conlleva a que las ciudades griegas no fuesen únicamente el resultado de la agrupación de demos distintos, sino también el resultado de la concentración moral y morfológica de formas de asociación anteriores y elementales (gens, filias, fatrias). Para Durkheim, la ciudad griega constituía un nuevo régimen que mitigaba y agregaba a los anteriores. En esta nueva configuración las formas de asociación precedente no quedaban sumadas, negadas ni superadas, sino que, enlazadas al nuevo conjunto, sin desaparecer o ser trastocadas en la nueva asociación y, de manera significativa, esta nueva disposición tenía también un registro material, por tanto la "morfología" a la que aludía Durkheim no era solo referida a la forma de las asociaciones como ocurre en Simmel (2014).

Menciones a la morfología social y a la configuración agregacional de las ciudades pueden ser encontradas en otras reseñas a textos referidos generalmente a la historia de las ciudades e instituciones griegas y romanas (Durkheim, 1975). También en Las formas elementales de la vida religiosa, publicado en 1912, Durkheim vuelve a mencionar la noción de una base morfológica material, vinculada, pero distinta de la conciencia colectiva (1912/2012). Posteriormente Maurice Hallwbachs (2004) fue quien desarrollaría con mayor profundidad tanto la noción de "morfología social" como su aplicación al estudio de los entornos urbanos, pero escasamente dedicaría atención al estudio específico de los entornos materiales o de las edificaciones dentro de las ciudades.

Durkheim tampoco desarrolla más estos temas, pero alude a ellos directamente en El suicidio. Estudio de sociología (1897) cuando señala que

... no es cierto que la sociedad solo esté compuesta de individuos; comprende también cosas materiales que juegan un papel esencial en la vida común. El hecho social se materializa en ocasiones hasta convertirse en un elemento del mundo exterior. Por ejemplo, un determinado tipo de arquitectura es un fenómeno social; ahora bien este tipo está encarnado 
en parte en las casas, en los edificios de toda clase que, una vez construidos, se convierten en realidades autónomas, independientes de los individuos... La vida social, que está de este modo como cristalizada y fijada sobre soportes materiales, se encuentra por eso mismo exteriorizada y actúa sobre nosotros desde afuera (Durkheim, 1897/2004, p. 427).

Para Durkheim un edificio, al igual que otros objetos materiales, es tanto una "expresión del estado de la técnica" (2004, p. 427) como un elemento morfológico que cristaliza un estado de lo social, un hecho técnico que tiene, por tanto, la capacidad de afectar la acción de los individuos que entran en interacción con él. Lo interesante dentro de las aproximaciones de este autor es que, independientemente de su nulo desarrollo posterior, da a las edificaciones (y a los objetos del mundo material) la posibilidad de ser objetos de estudio sociológico, a la vez que da a los "hechos sociales" la posibilidad de "cristalizarse", volverse materiales y, con ello, adquirir las cualidades de los materiales: la duración, la movilidad, la reparación, entre muchas otras posibilidades.

Cabe también considerar que la teoría de la dominación Max Weber puede aportar al estudio de las edificaciones, en particular los efectos que tiene el ordenamiento del espacio en las acciones de los individuos y que puede extraerse de su tipología de las ciudades (Weber, 2005). Ese texto en particular apareció en 1921 con el título de La ciudad en los Archiv für Sozialwissenschaft und Sozialpolitik, donde fue editado en su versión inglesa, pero fue integrado en la cuarta versión alemana con el título La dominación no legítima. Tipología de las ciudades, lo que derivó en que el análisis weberiano del espacio urbano fuese integrado al análisis de las formas de dominación junto con el análisis de la relación entre función económica y ciudad (Colom González, 2014; Martindale, 1966). Johannes Winckelmann, responsable de esta inclusión, argumenta que el dominio no legítimo surgió como consecuencia de la constitución de las ciudades como comunidades autónomas libres, es decir, sin la presencia de una autoridad legítima como fuente exclusiva de toda autoridad (Weber, 2005). Tales consideraciones acerca de la legitimidad del ordenamiento espacial son aplicables también a los programas internos de los edificios y su disposición en las ciudades.

Desde esta perspectiva las edificaciones y, dentro de ellas los edificios de altura, afectarían las decisiones (o sentidos) de acción de los individuos a través de la morfología de su espacio, mediante los requerimientos de obediencia, y en función de las normas, aunque no suponen de por sí ningún derecho para sus habitantes, no constituyen comunidad y son independientes de la constitución de una "autoridad legítima" (Weber, 2005, p. 966).

Lo anterior es de suma utilidad para el estudio del diseño y funcionalidad de las divisiones especiales internas de los edificios (programas) y de su capacidad agencial en las prácticas de los individuos, así como la presencia de dispositivos legales y territoriales que colaboran con la discusión acerca de si sus habitantes constituyen comunidades y/o son objetos de derechos (Colom González, 2014). En este punto, la teoría de los vínculos de Tönnies podría ser también de utilidad para trazar las formas en las que las diversas asociaciones entre individuos se enlazan en una configuración intermedia entre la residencia habitacional y la ciudad, y verificar si las asociaciones articuladas en ellas corresponden o no a comunidades y cuáles serían sus características.

A pesar de no abordar directamente las edificaciones como objetos de análisis, hay en la sociología temprana cierta preocupación por la relación entre el espacio y las interacciones que es posible utilizar para el estudio de la arquitectura de las ciudades, pero que no va más allá de la aplicación teórica. Los sociológicos de la fase temprana, salvo quizá Durkheim, abordaron los fenómenos urbanos como fenómenos abstractos $y$, dentro de ese plano, las evidentes variaciones arquitectónicas de la forma urbana o las diversas entidades técnicas que comenzaron a poblar las ciudades modernas (edificios de altura, automóviles, tranvías, entre otras) no captaron la atención sistemática de los practicantes tempranos de la sociología.

\section{El edificio de altura en la sociología urbana}

El Departamento de Sociología de la Universidad de Chicago fue fundado por Albion W. Small en 1892, tres años antes de que Emile Durkheim fundara el primer departamento en tierra europea, en 1895 en la 
Universidad de Burdeos, de modo que cabe dentro del periodo de consolidación institucional de la sociología (fase temprana). Sin embargo, la conjunción de pensamientos sociológicos que se conoce como la Escuela de Chicago y que resulta en una primera articulación del campo de estudios que se conocerá como sociología urbana es levemente posterior. Cuaja con la aparición de The city: Suggestions for Investigation of human behavior in the urban environment (Park \& Burgess, 1925), que no solo recoge textos que venían siendo publicados en revistas desde 1914, sino también establece un canon sociológico con una función específica: el estudio de la comunidad urbana (Wirth, 1925).

A partir de ello, el estudio de comportamiento de los individuos en los espacios urbanos, su mentalidad, la distribución espacial de las actividades urbanas y los patrones de crecimiento y planificación de las ciudades son abordados por una misma comunidad científica bajo un programa más menos común. Los temas de estudio de esta escuela, si se contemplan los publicados desde 1923, no consideran específicamente a los entornos materiales urbanos y menos las edificaciones. Los más próximos a esto son mapas de zonas de la ciudad de Chicago con datos sobre su ocupación de edificaciones elaborados por Ernest Burguess para el gobierno de la ciudad.

A pesar de que los trabajos de Anderson (1923), Cressey (1932), McKenzie (1923), Thrasher (2013) o Wirth (1998), describen vidas que transcurren entre los blocks, teatros y rascacielos de Chicago, pocas veces se detienen a dar noticias sobre ellos o a considerarlos como elementos que afecten especialmente a las personas o situaciones que detallan. Quizá uno de los pocos textos que entra en algunos detalles es The dweller in furnished rooms: An urban type de Harvey Warren Zorbaugh (1926) que describe el comportamiento y la personalidad de los arrendatarios de piezas amobladas en la zona del Lower North Side de Chicago.

El mismo Zorbaugh será uno de los primeros en darle protagonismo a los edificios de altura en su The gold coast and the slum: Sociological study of Chicago's Near North Side, publicado en 1976. Allí, mientras describe la vida entre el área central del Chicago (Loop), señala que
... el rascacielos es el símbolo visible del dominio del Loop sobre la vida de la ciudad. El distrito central de negocios de la ciudad antigua, al igual que en el Londres moderno, con sus edificios de seis y ocho pisos, se extendía sobre una zona difícil de manejar. Pero el rascacielos, que empuja el Loop hacia el cielo treinta, cuarenta, cincuenta pisos, ha hecho posible una extraordinaria centralización y articulación del distrito central la nueva ciudad. Atrayendo a miles de personas diariamente hacia el corazón de la ciudad, donde los viejos tipos de edificios solo podían atraer a unos cientos. El conjunto de rascacielos del Loop se ha transformado en el vórtice de la ciudad (Zorbaugh, 1976, p. 2).

Las descripciones de los rascacielos del Loop no son las únicas del texto, ya que estas se extienden a las barriadas (slums), a los edificios de oficinas, los edificios de arriendo, entre otros. De hecho, The gold coast and the slum... es uno de los primeros textos de sociología donde la observación de las formas arquitectónicas, sus tipologías, sus alturas y sus aglomeraciones ayudan a explicar las diferencias funcionales dentro la ciudad y la segregación dentro de esta. Zorbaugh distingue entre tipologías como rascacielos, edificios de oficinas, edificios de fábricas, los edificios residenciales, edificios de alta renta, y barriadas, entre otros. También ubica las edificaciones que acogen a cabarets, suicidios, entre otros eventos. En The gold coast and the slum... los edificios son protagonistas de la vida en Chicago, así como también datos en su estudio sociológico.

Como se verá, la sociología de Chicago hacia 1930 no era propiamente una materia acerca de "la ciudad", sino una disciplina respecto de lo que ocurría dentro de ella. Sus conceptos articuladores son los "ambientes", las "ecologías" y las "comunidades" urbanas (Anderson, 1960; Park \& Burgess, 1925) más que “lo urbano" en sí. Por esta razón, cuando Maurice Halbwachs, el discípulo de Durkheim quien hereda su preocupación por las ciudades y la temática de la morfología urbana visita Chicago, a pesar de la admiración que tiene por los trabajos y metodologías utilizadas por los miembros de esta escuela, no ve puntos de conexión entre su trabajo y el de los de Chicago (Farfán, 2004; Martínez Gutiérrez, 2008), salvo quizá con el de Burgess respecto del crecimiento de las ciudades. 
En una línea opuesta a Zorbaugh, Halbwachs (1932) en Chicago verá una ciudad inestable materialmente, no consolidada, con grandes espacios vacíos donde poblaciones flotantes se instalan y se marchan con facilidad. Una ciudad de surgimiento intenso, constante y extensamente en construcción, que, "a finales de 1830 ... no había allí más que un pueblo, que comprendía doce casas y dos residencias suburbanas, quizá con unos 100 habitantes" (p. 200), pero que para 1930 contaba con casi tres millones y medio de habitantes. Trayectoria muy distinta al París centenario adaptado lentamente por Haussmann, o al Berlín, igualmente añoso, que creció agregando a sí los poblados cercanos. La diferencia entre estas y otras ciudades señalará Halbwachs luego de la comparación, radica en la "rapidez desigual de su formación" (1939, p. 295), puesto que las ciudades son finalmente fenómenos poblacionales y, por tanto, el eje de su estructura morfológica es la densidad poblacional (Halbwachs, 1938).

Es claro que también otras densidades se aglomeran en las ciudades, pero a ellas Halbwachs no les asigna la misma importancia que a las densidades poblacionales, aunque se trate de los rascacielos de Chicago. Es en un texto posterior, La memoria colectiva (Halbwachs, 2004), donde aborda la materialidad, las edificaciones y los lugares de las ciudades bajo el punto de vista de su valor espacial y simbólico para sus habitantes. Fiel a la escuela durkheimiana, Halbwachs verá en ellos cristalizaciones de lo que los colectivos humanos quieren recordar en el espacio.

Con todo, Halbwachs es el primer antecedente de una escuela de sociología urbana en Francia (Ullán de la Rosa, 2014). Le siguen, entre otros, Paul Henry Chombart de Lauwe y Raymond Ledrut, funcionalistas que harán de los edificios residenciales de la escuela moderna parte de su objeto de estudio. Las investigaciones desarrolladas por Chombart y Ledrut desde la década de 1950 ocurren en conjuntos habitacionales que superaban los cinco pisos de altura, edificaciones estatales destinadas a los segmentos medios y de trabajadores especializados (Busquet, 2014). Estos estudios, basados en datos cuantitativos recogidos en los edificios, evaluaban (ya no describían) la densificación poblacional (Chombart de Lauwe, 1952), la sociabilidad en los barrios y las unidades vecinales (Chombart de Lauwe, 1977), la satisfacción de los habitantes con la vivienda y con el equipamiento urbano (Chombart de Lauwe, 1959) y los desplazamientos de los habitantes dentro de la ciudad (Chombart, 1952; Ledrut, 1974).

Tales temáticas se consolidan especialmente en torno a los problemas de la vivienda y vida cotidiana en las ciudades (Merton, West, Jahoda \& Selvin, 1963; Ullán de la Rosa, 2014) y se reflejan en conjuntos de edificios de altura diseñados bajo una impronta de habitar colectivo y serializado (Guillén, 2006), pero las variables propiamente arquitectónicas solo son tomadas en consideración como contextos o escenarios de comunidades e individuos, y no como elementos que constituyan en sí un objeto de estudio sociológico.

Henri Lefebvre cambia eso al considerar al espacio como una "relación social" y desplazar el objeto de estudio desde las comunidades urbanas hacia el espacio mismo, bajo el argumento de que, al igual que el capital, este es capaz de articular un modo de producción particular (Lefebvre, 1971; 2013). De esta forma, el espacio también desplaza a la ciudad como objeto de estudio, puesto que esta no es más que el resultado de la producción del espacio y que el sentido productivo de este espacio, "lo urbano", tampoco podía ser contenido en los límites de la ciudad, sino que tendía a escalar hacia el globo, subsumiendo también lo restante de la vida agraria o rural (Lefebvre, 1971; 2013).

A diferencia de la ciudad, sostiene Lefebvre (1971), "lo urbano" no es un objeto claramente definido ni definitivo, no es un objeto para una ciencia o una meta inmediata para la acción, sino un objeto virtual, posible. "Lo urbano" se define como horizonte y como virtualidad clasificadora, como abstracción científica de una espacialidad donde se cancelan las distancias, mientras que su problemática se define en las diferencias de acceso a la sociedad urbana, y las implicaciones y consecuencias de estas.

Dentro de esto, las formas arquitectónicas son solo relevantes en un nivel agencial, en el mismo sentido utilizado por Durkheim, cristalizan o adquieren forma de relaciones sociales que no son explicadas por ellos mismos, sino por la función que despliegan. Para Lefebvre, el edificio "tiene una función, una forma, una estructura, pero no reúne todos los momentos formales, funcionales y estructurales de la práctica social" (2013, p. 265), por ello le atribuye un desempeño similar al del sintagma en relación con la producción del espacio. Los 
edificios, efectúan "sintagmáticamente" la vinculación de las actividades en el espacio social como tal, es decir, en el espacio económicamente administrado por el capital, socialmente dominado por la burguesía y políticamente organizado por el Estado.

De esta manera, el edificio, en un sentido genérico ${ }^{2}$, constituye una forma particular de organizar el espacio que condensa (o refleja) los atributos de la sociedad, desplaza las actividades, las localiza y las hace viables por medio del equipamiento. Resulta así una práctica espacial, una representación del espacio y un espacio de representación, al mismo tiempo (Lefebvre, 2013, p. 92).

El giro hacia el espacio de Lefebvre, y el consecuente desplazamiento de las comunidades y la ciudad como objetos de estudio, permitía "seguir" la producción del fenómeno observado a través de objetos diferentes, como piezas, casas, edificios y ciudades, e independiente de su ubicación rural o urbana, sin embargo, también tenía la capacidad de diluir a la sociología urbana, lo que motivó inmediatas críticas.

Una de ellas, provino de un cercano de Lefebvre, Manuel Castells, quien cuestionó tanto el sentido dado al espacio por Lefebvre como la metodología utilizada por este, ya que ofrecía "una teoría de la producción del espacio sobre una base estrictamente filosófica, sin un profundo conocimiento de los datos económicos y tecnológicos sobre los procesos de urbanización y sobre su organización social y política" (Pickvance, 2007).

Para Castells (1974) el "espacio" es una expresión de la estructura social y no una variable que pudiese explicar por sí misma las relaciones y prácticas sociales que se desarrollan en una sociedad. Por el contrario, lo relacional predomina sobre lo físico en la conformación de la práctica social, lo cual desaconseja considerar a las ciudades como una variable independiente capaz de explicar las formas de las relaciones sociales. Como

\footnotetext{
${ }^{2}$ El término 'edificio', en Lefebvre, designa una "arquitectónica del espacio" en particular, una forma de organización del espacio social asociada a una función. Para el autor, esta "matriz homogénea del espacio capitalista, el edificio, en su predominio, sabe combinar el objeto de control por el poder y el objeto de intercambio comercial. Procede mediante la condensación brutal de las relaciones sociales ... el edificio cubre, reduciéndolo, el paradigma del espacio: dominaciónapropiaciónn (privilegiando la dominación tecnológica); obra y producto (privilegiando el producto); inmediato y mediación (privilegiando las mediaciones y a los mediadores, del instrumental técnico a los "promotores" financieros de las operaciones constructoras). Reduce las oposiciones y valores significativos, entre otros los del goce y el sufrimiento, los del uso y del trabajo (2013, p. 269).
}

explica Sharon Zukin (1980) esto implica metodológicamente que si las ciudades solo reproducen o reflejan las contradicciones de una estructura social dada, su estudio es equivalente al de la sociedad en su conjunto y, por tanto, careciendo de especificidad, se tornan irrelevantes en tanto objeto de estudio.

Castells dedica varios textos para esta demostración. Comienza en 1968 con “¿Existe la sociología urbana?” (Pickvance, 2007, pp. 33-59), que es entendido en su momento como un desafío coyuntural pero que luego, en Problemas de investigación en sociología urbana, adquiere el carácter de un intento sistemático por provocar un giro radical en el campo de estudios que terminará por diluirlo (Elliott, 1980; Gottdiener \& Hutchison, 2011; Lebas, 1982; Zukin, 1980; 2011). Es en este último texto donde tanto traza una historia disciplinar y un canon de la sociología urbana ${ }^{3}$, como también desestima a la "cultura urbana" como su objeto de estudio, señalando que no es otra cosa que un mito y que esta carece de objeto de estudio real (Castells, 1971).

Como reacción a este giro crítico, nuevas generaciones de sociólogos buscaron reafirmar lo urbano utilizando sus conflictos como objeto de análisis e integrando enfoques explicativos de estos provenientes de otras disciplinas como la economía, la geografía y la ciencia política (Hashimoto, 2002; Lussault, 2015; Milicevic, 2001; Perry \& Harding, 2002). Este esfuerzo, conocido como New Urban Sociology, puso énfasis en el análisis del espacio, su distribución y los conflictos originados en él, considerando que reflejaban las contradicciones propias del estado del capitalismo (Gottdiener, 1994). Son estas contradicciones y los conflictos que emergían de ellas las que capturan la atención en estos estudios, indudablemente inspirados en los trabajos de Jane Jacobs (1961), y sus objetos pasan a ser los individuos y colectivos que protagonizan las controversias y conflictos, no los entornos materiales o arquitectónicos. Para esta nueva sociología urbana los edificios vuelven a ser invisibles.

Este nuevo giro permitió sumar al activismo político con el estudio de lo urbano y del conflicto social en las ciudades, conformando una agenda amplia que abordaba

\footnotetext{
3 De la cual separa a los “Community Studies" (Lefebvre, 1971, p. 47), cuya fecha de despegue puede ser adosada al canon publicado por Louis Wirth en The city (Park \& Burgess, 1925).
} 
también los fenómenos relacionados con el poder político y administrativo en torno a la planificación, el acceso y control de recursos, el consumo, la distribución de bienes y servicios y la globalización de lo urbano (Gottdiener \& Budd, 2005; Gottdiener \& Hutchison, 2011). El efecto fue "escalar" aún más la observación y profundizar la distancia entre los estudios centrados en lo urbano y las ciudades, permitiendo abordar los fenómenos relacionados con la globalización de lo urbano y de sus infraestructuras (Sassen, 1991; 2006), pero siguiendo esta vez el rastro de los conflictos, no de las comunidades, de la materialidad o del espacio (Brenner, 2009).

Sin embargo, para el coloquio organizado en 2001 por las asociaciones británica y norteamericana de sociología, no se llegaba a la conclusión de que la sociología urbana tuviese un objeto de estudio específico y viable, sino que más bien la opinión general seguía siendo que se trataba de un campo de estudios que respondía a una multiplicidad de temáticas y diálogos con otras disciplinas, donde los conceptos como el espacio, la comunidad y lo urbano se tornaban abiertos y porosos (May, Perry, Galès, Sassen \& Savage, 2005; Perry \& Harding, 2002). La duración de esta constante inestabilidad en los objetos de estudio ha afectado obviamente la identidad de la sociología urbana, en tanto subcampo, pero no ha detenido el interés disciplinar por estudiar elementos identificables con las edificaciones y las ciudades, toda vez que ambos elementos se tornan críticos para el habitar humano.

\section{El edificio de altura en el giro hacia las espacialidades}

Como se señaló anteriormente, quizá el aporte más relevante de Lefebvre, en pos de la relación entre la sociología y los edificios de altura y/o el estudio sociológico de las edificaciones y la arquitectura, es haber desplazado del centro del análisis a los actores humanos (comunidades), así como haber puesto en su lugar una relación social, un actor no humano, una forma técnica. La diferencia entre uno y otro radica en que los actores humanos (comunidades) constituyen un centro centrífugo, su diversidad de intereses y la complejidad de estos hacen que, a pesar de ocupar el centro de la observación, a la larga la redirijan hacia fuera de ellos.
Como decía Durkheim, la explicación de un hecho social no está en él, sino en otro(s) hecho social.

El actor no humano, en cambio, la forma técnica, la relación social o el objeto, deben ser determinados externamente, requieren asociar las cosas que los definen $y$, en lo posible, mantenerlas unidas y ello hace que, tomados como centros, motiven fuerzas centrípetas. El resultado de esto no solo es un dibujo donde las cosas convergen en vez de ser expulsadas desde el centro, lo que converge lo hace desde distintas posiciones y con trayectorias diferentes; la convergencia implica acercar dichas posiciones, llegar a negociaciones que parten desde una diferencia basal.

Al colocar al "espacio" en el centro de la observación, Lefebvre posibilitó una dinámica convergente, pero ello fue opacado por la discusión acerca de "lo urbano", que devino en una dinámica centrífuga que terminó diluyendo el subcampo de estudios que se trataba de organizar. Un ejemplo de esto es lo que se aprecia respecto de los edificios en Lefebvre. Lo que le permite hablar acerca de ellos es el "espacio", no "lo urbano"; por el contrario, "lo urbano" torna invisible no solo a los objetos de arquitectura, sino a toda la escala de observación en que estos se constituyen.

Sin embargo, con la crisis "lo urbano" -que no es otra cosa que el efecto de las fuerzas centrífugas que terminan expulsando los objetos de estudios hacia disciplinas colindantes-, "lo espacial" volvió a brillar y a convocar nuevamente a los investigadores (Martínez López, 2005). Por cierto, ya no se trataba de un objeto unívoco (el espacio), sino más bien un objeto múltiple y heterogéneo (espacialidades), que ya no podía ser fijado en un solo punto y que asume distintas formas o modos de existir socialmente.

Una de estas formas de espacialidad es el "lugar", que ha adquirido peso como variable de observación para explicar la movilización de actores y tendencias socioculturales. En torno a ello se han desarrollado dos tipos de discusiones. Aquella que otorga al "lugar" un valor extraíble (bien) y cuestiona las formas de extracción, distribución y apropiación de dicho valor, en cuyo entorno se alojan las temáticas como el patrimonio urbano, comunes urbanos y derecho a la ciudad (Borch \& Kornberger, 2015; Brenner \& Theodore, 2005; Brenner, Madden \& Wachsmuth, 2011; Harvey, 1992). Y aquella que otorga al "lugar" un valor constitutivo (territorio) y 
verifica las formas como este se organiza, distribuye y apropia, con atención a la especificidad empírica y pertinente a los asuntos de gobierno (Hall, 2015; Sassen, 2005; Savage, 2005).

Si en el primer caso, el "lugar" asume el rol de un recurso cuya gestión entra en discusión, en el segundo toma un rol constituyente cuya activación y distribución son las que entran en cuestión. En ambos casos, los edificios solo se constituyen en objetos de estudio (solo constituyen "lugares") cuando tienen relación con los espacios como recursos extractivos o como elementos constituyentes, lo cual los vincula en otras temáticas, ya sea describiendo los fenómenos de asociatividad y colectivismo que se desarrollan al interior de edificios residenciales (McGuirk, 2014; Venkatesh, 1997; 2008; 2013; Yau, 2011; 2012), o describiendo la densificación urbana a través de ellos ( $\mathrm{Ng}, 2010)$.

Como ya se señaló, ambas discusiones son posibles de escalar hacia las ciudades, que en la escala subglobal adquieren mayor sentido espacial que los países que son, en esta escala, el tipo de "lugar" mayormente apelado por los estudios (Brenner \& Schmid, 2014; Burdett \& Sudjic, 2007; McGuirk, 2014; Sassen, 1991; 2005; Sennet, 1990; Venkatesh, 2013). Ya sea como "recurso" o como "territorio", la espacialidad conlleva una constitución técnica, se presenta como un objeto técnico que para su explicación sociológica recurre a instrumentos y parámetros técnicos de otras disciplinas, como la economía y la ciencia política.

Otra forma de espacialidad es la que la considera como el efecto de un "ensamblaje sociotécnico", como un objeto complejo donde la tecnicidad del espacio proviene del conjunto heterogéneo de actores humanos y no humanos que se enlazan en él y le constituyen. Este tipo de aproximaciones provienen de los estudios de Ciencia, Tecnología y Sociedad (CTS), que centran su observación en los objetos técnicos y las prácticas que estos motivan por lo mismo, dentro de este campo de estudios, las espacialidades constituyen un tipo particular de ensamblaje, ya sea ecológico (Star \& Griesemer, 1989), de objetos (Blečić \& Cecchini, 2017; Guggenheim, 2009) y/o un tipo especial de tecnología (Gieryn, 2002; Guggenheim, 2010).

A pesar de no haber "sufrido" la crisis de la sociología urbana, el enfoque sociotécnico también se resuelve de una manera multidisciplinar en dos sentidos: se nutre de los avances y conclusiones de otras disciplinas y asume los lenguajes de las disciplinas asociadas a los objetos de estudio (arquitectura, urbanismo, ingeniería) como parte del campo observado (Callon, 1984). A su vez, también asume una condición política $y$, aunque no es tan marcadamente asociada al activismo urbano, sí lo es respecto de los problemas de gubernamentalidad (Brenner et al., 2011; Farías \& Bender, 2010; Yaneva, 2017).

Todo lo anterior implica un cálculo de gobernanza que armoniza dentro de estas entidades distintos regímenes técnicos, distintas prácticas y distintos intereses propios de los actores que son convocados a constituir asociaciones con estos objetos. Uno de los énfasis defendidos en este tipo de estudios es que estos problemas son contingentes en todas las etapas de vida de las edificaciones y que las controversias en torno a las edificaciones son constantes y persistentes, de forma que su proceso constitutivo nunca está completamente cerrado, algo que también es sostenido en textos arquitectónicos como How building learn de Steward Brand (1994) o ¡El tiempo construye! de GarcíaHuidobro, Torres y Tugas (2008).

Dentro de esta perspectiva Thomas Gieryn (2002) ha sostenido que los edificios pueden comportarse como artefactos tecnológicos y/o como objetos materiales, producto de la agencia humana y como una fuerza estable de la acción de la estructura social, aspecto que también había sido discutido por Langdom Winner respecto de la "capacidad política" que tenían los puentes de Nueva York (Joerges, 1999; Winner, 1980; Yaneva, 2017). Bruno Latour (1987), por otro lado, a razón de su estabilidad locacional, de su singularidad objetual y de los modos específicos de su uso (que son cambiantes), propone que los edificios se comportarían de forma diferente a otros objetos (inmutables móviles), como "mutables inmóviles" (Guggenheim, 2010).

Para Latour (1987), por ejemplo, mientras más modernas y complejas son las máquinas, más formularios necesitan para nacer, porque es más difícil trazar sus partes y es más probable que los aspectos no trazables se tornen en "cajas negras". Algo similar ocurre con los edificios, antes de ser construidos estos se calculan, se dibujan, se escriben y se discuten, pero también en su proceso de construcción y, sobre, todo, también durante su funcionamiento cotidiano. Lo que ocurre con la 
enorme producción de dispositivos, informes, manuales, señaléticas, entre otros, no solo es que se van acumulando, sino también van haciendo trazables las situaciones por las que atraviesa el edificio, las asociaciones en las que es incluido, los actores con los que interacciona, las controversias que se producen. Todo elemento presente tiene un manual, un formulario que lo llevó hasta allí y otro que lo hace permanecer. Facturas, contratos, actas y memorándums, constituyen centros de cálculo cada vez más heterogéneos que proliferan con los objetos al interior del edificio, expanden su gubernamentalidad, materializan sus vínculos como una red sociotécnica.

Por otro lado, bajo la consideración simétrica de entidades tecnológicas, la resolución de cada controversia mediante un nuevo conector, mediante un nuevo dispositivo normativo o mediante el ingreso o salida de actores, obliga a considerar que, como entramado de entidades, el edificio no solo resulta un ensamblaje (Guggenheim, 2010), sino también un tejido (una entidad material) capaz de crecer y decrecer (Ingold, 2008).

Premisas similares han sido utilizadas en la observación de los edificios bajo una perspectiva sociotécnica que han verificado configuraciones estables de asociaciones y efectos interaccionales entre actores humanos y no humanos. Entre las temáticas abordadas por estos estudios se cuentan: la tipología misma de edificios de altura (Graham, 2016; Grubbauer, 2010; Kuppinger, 2010); el funcionamiento de edificios individualizados como laboratorios (Gieryn, 2002; 2008), hospitales (Chang, 2010), museos (Beauregard, 2015; Hancock, 2010; Yaneva, 2005; 2008), iglesias (Edensor, 2011), parlamentos (Dányi, 2015); o como instrumentos geopolíticos (Graham, 2004), las prácticas relacionadas con su diseño arquitectónico (Fallan, 2008; Müller \& Reichmann, 2015); los efectos interaccionales (morales) de estos diseños (Chan, 2015); o los motivos y resultados de los cambios de programas de funcionamiento dentro de estas formas arquitectónicas (Guggenheim, 2010).

A diferencia de las investigaciones que privilegian el enfoque de "lugar" en las edificaciones, que buscan verificar y explicar el efecto de una variable de naturaleza externa a las edificaciones, este tercer cuerpo de estudios no busca explicar cómo son construidos socialmente los edificios, sino contestar qué es lo que este particular tipo de objeto técnico explica acerca de los actores y sus asociaciones, comunicar las múltiples y heterogéneas formas en las que el mundo se ordena y desordena (Law, 2002). Esto conlleva a un programa analítico que pone en primer plano las dimensiones instrumentales, performativas y materiales implícitas en la elaboración de hechos, objetos $y$, en este caso, edificios.

A pesar de sus aportes, el riesgo de esta perspectiva radica en sobredimensionar las situaciones agenciales (ensamblajes, asociaciones) y dejarlas desenlazadas de las de orden estructural en las que también participan, descuidando la explicación acerca de la capacidad práctica del actor para ser coherente en diversos planos o escalas de interacción y/o sobre las prácticas a desarrollar para mantenerse y hacer durar las asociaciones en las que participa (Callon, 1984). El peligro latente en esto es quedarse en la contabilidad de las asociaciones de actores convocados, sin enlazarlas hacia escalas diferentes de la observación agencial.

\section{Conclusiones}

A lo largo de su desarrollo la sociología ha explorado distintas aproximaciones para determinar qué observar cuando se trata de las ciudades, de manera de entender los procesos que ocurren en ellas y que la implican disciplinariamente. Todas las discusiones relatadas tienen su origen en diferencias de corte epistemológico, varían según el punto de observación del fenómeno y la forma en que este enlaza a una explicación acerca de su relación con el mundo. Estas variaciones son radicales en el caso de los objetos arquitectónicos y en espacial en el de los edificios de altura.

Atendiendo a esto, si la sociología temprana, a pesar de sus evidentes aportes, careció completamente de una intención de organizar un subcampo de estudios que tuviese como centro la arquitectura y sus edificaciones, las diversas escuelas que organizaron el subcampo urbano compartieron esa falta de intensión con el problema adicional de no lograr estabilizar tampoco entre ellas, el punto cero del subcampo. Si en la Escuela de Chicago se discutía entre poner en el centro a las comunidades urbanas o al comportamiento de los individuos en las ciudades, en la Escuela Urbana Francesa se discutió si el centro eran los habitantes o el espacio, en tanto relación social. Finalmente, la nueva sociología 
urbana, al ver que lo urbano escalaba hacia lo global y se diluía como objeto específico, propuso a los conflictos y activismos urbanos como centro del subcampo, obteniendo el mismo efecto anterior: lo urbano resultaba explicado por algo externo a él y resultaba, como tal, irrelevante.

En ninguno de estos casos fue posible articular un subcampo con centro en la arquitectura y las edificaciones a pesar de que, como hemos visto, existieron intentos de considerarlas en más de una ocasión. Sin embargo, dentro de la situación de crisis en la sociología urbana, comenzó a tomar forma una nueva centralidad, insuficiente para organizar en torno suyo al conjunto de estudios urbanos, pero capaz de retomar el giro espacial iniciado por Lefebvre, lo cual implica un centro conceptual con la habilidad de enlazar los problemas de escala, es decir, capaz de organizar el estudio de una habitación, un edificio, una ciudad y lo global: la(s) espacialidad(es).

Sin embargo, también en ello hay discusión. En lo anterior solo se describieron tres grandes líneas por las cuales se están desarrollando estudios con foco en espacialidades y probablemente con el tiempo se generen muchas más. Lo importante respecto de esto son dos cosas: los estudios en espacialidades, a diferencia de los esquemas anteriores, operan con una lógica centrípeta respecto de su objeto de estudio; y, segundo, este objeto de estudio tiene la cualidad de poder ser observado en diferentes escalas, incluso es posible observar su desplazamiento entre escalas, como ocurre con los mapas actuales, de forma que también ofrece una posible salida a dos problemas sociológicos clásicos: la oposición entre agencia y estructura, que sería irrelevante si es posible trazar con claridad los caminos entre ellas; y la dualidad entre agencia y estructura, que se demostraría ineficiente heurísticamente si se identifican escalas intermedias.

Es la relación con el problema de las escalas de observación, que caracteriza este nuevo objeto de estudio, lo que parece ser el aspecto más importante del giro hacia el espacio dado por Lefebvre y que consolidan las diferentes líneas de estudios sobre espacialidades. Tanto los enfoques de "lugar" como los enfoques objetuales (o ensamblajes) apelan a espacialidades que pueden enlazarse multiescalarmente. Ocurre con las ciudades en el caso de los trabajos de Sassen, Brenner y otros del enfoque de "lugar", que son capaces de enlazar barrios, países y escenarios globales; y ocurre con los edificios en los trabajos de Brand, Guggenheim y Yaneva, entre otros del enfoque objetual, que enlazan intereses de actores de diversa escala (individuos, instituciones locales, nacionales y globales).

El protagonismo que adquieren los enlazamientos entre escalas, presentes en los estudios con enfoque en las espacialidades, pone de relieve otro fenómeno que no había sido considerado como variable en los enfoques anteriores: las prácticas de coordinación entre actores que son necesarias para articular y sostener el enlazamiento entre espacialidades y/o el tránsito de estas entre escalas entre otras posibles necesidades. Ese aspecto no fue visto por la sociología temprana y tampoco por la sociología urbana en lo que respecta a sus objetos de estudio, y evidencia que estas nuevas aproximaciones colaboran también con la identificación de nuevos problemas.

El caso los edificios de altura, como objetos estudios sociológico, tiene que ver con el giro antes descrito. Son más claros de ver cuando el fenómeno central son las espacialidades y una prueba de ello es el creciente número de textos que, dentro de estos enfoques, son dedicados a ellos. En estos, los edificios aparecen como "lugares" donde encuentran agencia fenómenos estructurales como la segregación, la gentrificación, la reproducción de los modelos familiares, entre muchos otros, y ello ocurre porque estos "lugares" colaboran activamente con ello. Por eso hay que demolerlos si se quiere solucionar el problema como ocurre en los casos narrados por Venkatesh en Chicago $(1997 ; 2008)$ o como ocurre en Bajos de Mena, en Santiago de Chile. En ese contexto, los edificios adquieren un protagonismo inédito, son tratados como una configuración espacial particular que tiene capacidad de producir resultados particulares (integración o segregación, por ejemplo).

Los edificios de altura también se muestran como objetos complejos, que no solo aumentan en número y diversidad dentro de las ciudades, alterando el curso de sus vientos, la temperatura de las calles, acogiendo mayores poblaciones, aumentado la carga de alcantarillado y cambiando el paisaje urbano, sino que también se tornan visibles cuando sus componentes fallan y centenares de personas pueden quedar "atrapadas" en ellos por un fallo en el suministro de 
electricidad, el mal funcionamiento de sus ascensores o inundaciones de cámaras producto de roturas de ductos. Entonces, estos objetos evidencian requerir de actores y prácticas de mantenimiento y cuidado permanentes que aseguren su funcionamiento constante y su persistencia, todo para que la espacialidad siga funcionando como tal.

Aspectos como los relatados no habían sido considerados por la observación sociológica debido a que la arquitectura, los edificios y los objetos urbanos en general no estaban incluidos en las diversas asociaciones urbanas analizadas por la disciplina, las que se habían acotado a las compuestas por actores humanos. Lo que ha permitido el giro hacia las espacialidades es complejizar la composición de estas asociaciones, integrando objetos técnicos de diversos tipos (uno de ellos, el mismo espacio) en el estudio de las asociaciones urbanas.

Este enfoque sociológico puede tener utilidad para abordar los efectos que tiene la proliferación de edificios de altura en las ciudades chilenas, no solo desde el punto de vista del aumento de su número o de su impacto en la composición material de las ciudades, sino también desde las asociaciones que convocan, los regímenes sociomateriales que despliegan, las controversias que se desarrollan en su interior y en su entorno y desde el punto de vista de las vulnerabilidades que implican a las ciudades.

Actualmente hay puntos ciegos en varias de las áreas señaladas donde, por ejemplo, se desconoce su número y su efecto en la densidad poblacional de las comunas,

\section{Referencias}

Adler, G., Brittain-Catlin, T., \& Fontana-Giusti, G. (Eds.) (2012). Scale: Imagination, perception and practice in architecture. London: Routledge.

Anderson, N. (1923). The Hobo. The sociology of the homeless man. Chicago: The University of Chicago Press.

Anderson, N. (1960). Sociología de la comunidad urbana. México: Fondo de Cultura Económica. no se sabe cuánto varían sus regímenes sociomateriales internos y las controversias debido a su proliferación o a sus prácticas de mantenimiento y cuidado han ido en aumento. Pero además, hay preguntas esenciales que también debiesen ser formuladas como aquellas que son relativas a las decisiones inscritas en sus diseños espaciales y los efectos biopolíticos de estos en la sociedad y en la gobernanza urbana.

Las edificaciones, y dentro de ellas los edificios de altura, no son solo la inscripción material de intereses y prácticas sociales que son movilizadas materialmente, no son objetos cerrados o finalizados en su constitución y en sus posibilidades de interacción, por el contrario, son reinterpretados y reorganizados constantemente, de forma que están permanentemente abiertos a la realización de lo social. De ahí la importancia de su observación sociológica.

A la luz de la revisión anterior es posible considerar que, a pesar de la casi nula atención que se les prestó en la sociología temprana y en las escuelas de sociología urbana, hay elementos en esas tradiciones que son útiles de considerar en los estudios actuales, sobre todo los que tienen que ver con la composición y organización de las comunidades que habitan en ellos y la legitimidad de su ordenamientos espaciales, pero cabe también considerar que el giro comenzado por Lefebvre tiene todavía mucho desarrollo por delante y el estudio de las edificaciones de altura representa una oportunidad única para profundizarlo [B]

Beauregard, R. (2015). We blame the building! The architecture of distributed responsibility. International Journal of Urban And Regional Research, 39(3), 533549. http://dx.doi.org/10.1111/1468-2427.12232

Blečić, I. \& Cecchini, A. (2017). On the antifragility of cities and of their buildings. City, Territory and Architecture, 4(1). http://dx.doi.org/10.1186/s40410016-0059-4 
Borch, C. \& Kornberger, M. (2015). Urban commons: Rethinking the city (space, materiality and the normative). London: Routledge.

Brand, S. (1994). How building learn. New York: Viking Press.

Brenner, N. (2009). What is critical urban theory? City: Analysis of urban trends, culture, theory, policy, action, 13(2-3), 198-207.

http://dx.doi.org/10.1080/13604810902996466

Brenner, N. \& Theodore, N. (2005). Neoliberalism and the urban condition. City, 9(1), 101-107. http://dx.doi.org/10.1080/13604810500092106

Brenner, N., Madden, D. J., \& Wachsmuth, D. (2011). Assemblage urbanism and the challenges of critical urban theory. City, 15(2), 225-240.

http://dx.doi.org/13604813.2011.568717

Brenner, N. \& Schmid, C. (2014). The 'urban age' in question. International Journal of Urban and Regional Research, 38, 731-755.

http://dx.doi.org/10.1111/1468-2427.12115

Burdett, R. \& Sudjic, D. (Eds.) (2007). The endless city: An authoritative and visually rich survey of the contemporary city. London: LSE Cities - Phaidon Press.

Busquet, G. (2014). La sociología urbana francesa y la evolución de las políticas urbanas de los años 1960 a los años 1980: ¿Porosidades, impermeabilidades o anidades electivas? EMPIRIA. Revista de Metodología de Ciencias Sociales, 27(enero-abril), 121-136.

http://dx.doi.org/10.5944/empiria.27.10865

Callon, M. (1984). Some elements of a sociology of translation: Domestication of the scallops and the Fishermen of St Brieuc Bay. The Sociological Review, 32(1), 196-233. http://dx.doi.org/10.1111/j.1467954X.1984.tb00113.x

Castells, M. (1971). Problemas de investigación en sociología urbana. México: Siglo XXI Editores.

Castells, M. (1974). La cuestión urbana. México: Siglo XXI Editores.
Chan, J. (2015). Moral agency in architecture? The dialectics of spatializing morality and moralizing space. En A. L. Müller \& W. Reichmann (Eds.), Architecture, materiality and society. London: Palgrave Macmillan.

http://dx.doi.org/10.1057/9781137461131_10

Chang, J. H. (2010). Tropicalizing technologies of environment and government: The Singapore General Hospital and the Circulation of the Pavilion Plan Hospital in the British Empire, 1860-1930. En M. Guggenheim \& O. Söderström (Ed.), Re-shaping cities. How global mobility transforms architecture and urban form (123-141). London: Routledge.

Chombart de Lauwe, P. H. (1952). Paris et l'agglomération parisienne: L'espace social dans une grande cite. Paris: Presses Universitaires de France.

Chombart de Lauwe, P. H. (1959). Famille et habitation. Sciences humaines et Conceptions de l'habitation. Paris: Centre National de la Recherche Scientifique.

Chombart de Lauwe, P. H. (1977). La Vie quotidienne des familles ouvrières. Paris: Centre National de la Recherche Scientifique.

Colom González, F. (2014). Max Weber y la ciudad. Una interpretación a la luz de la experiencia hispanoamericana. En A. Morcillo \& E. Weisz (Eds.), Max Weber: una mirada iberoamericana (413-440). México: Fondo de Cultura Económica.

Contreras, Y. (2011). La recuperación urbana y residencial del centro de Santiago: nuevos habitantes, cambios socioespaciales significativos. EURE (Santiago), 37(112), 89-113. https://dx.doi.org/10.4067/S025071612011000300005

Contreras, Y. (2017). De los "gentries" a los precarios urbanos: los nuevos residentes del centro del Santiago. EURE (Santiago), 43(129), 115-141. https://dx.doi.org/10.4067/S0250-71612017000200006

Crang, M. \& Thrift, N. (Eds.) (2000). Thinking space. London: Routledge.

Cressey, P. G. (1932). The Taxi Dance Hall. A sociological study in commercialized recreation \& city life. Chicago: The University of Chicago Press. 
Dányi, E. (2015). The Parliament as a High-Political Programme. En A. Müller \& W. Reichmann (Eds.), Architecture, materiality and society: Connecting sociology of architecture with science and technology studies (pp. 99-118). London: Palgrave Macmillan UK

Delfante, Ch. (2006). Gran historia de la ciudad. De Mesopotamia a Estados Unidos. Madrid: Adaba Editores.

Durkheim, E. (1897/2004). El suicidio. Estudio de sociología. Madrid: Editorial Losada.

Durkheim, E. (1975). Textes III. Fonctions sociales et institutions. Paris: Les Éditions de Minuit.

Durkheim, E. (1912/2012). Las formas elementales de la vida religiosa. El sistema totémico en Australia (y otros escritos sobre religión y conocimiento). Buenos Aires: Fondo de Cultura Económica.

Edensor, T. (2011). Entangled agencies, material networks and repair in a building assemblage: The mutable stone of St Ann's Church, Manchester. Transactions of the Institute of British Geographers, 36, 238-252. http://dx.doi.org/10.1111/j.1475$\underline{5661.2010 .00421 . x}$

Elliott, B. (1980). Manuel Castells and the New Urban Sociology. The British Journal of Sociology, 31(1), 151158. http://.dx.doi.org/10.2307/590099

Engels, F. (1965). La situación de la clase obrera en Inglaterra (1845). Buenos Aires: Editorial Futuro.

Engels, F. (1976). El problema de la vivienda. Madrid: AKAL.

Fallan, K. (2008). Architecture in action: Traveling with actor-network theory in the land of architectural research. Architectural Theory Review, 13(1), 80-96. http://dx.doi.org/10.1080/13264820801918306

Farfán, R. H. (2004). Presentación. Un durkheimiano en Chicago: Maurice Halbwachs. Reis, 10, 201-213. http://dx.doi.org/10.2307/40184659

Farías, I. \& Bender, T. (2010). Urban assemblages. How actor-network theory changes urban studies. London: Routledge.
Friedrich, C. J. (Ed.) (1929). Alfred Weber's theory of the location of industries. Chicago: The University of Chicago Press.

García-Huidobro, F., Torres, D., y Tugas, N. (2008). iEl tiempo construye! Barcelona: Gustavo Gili.

Gehl, J. (2011). Life between buildings. Using public space. Washington, DC: Island Press.

Gieryn, T. (2002). What buildings do. Theory and Society, 31(1)5, 35-74. Recuperado de http://www.jstor.org/stable/658136

Gieryn, T. (2008). Laboratory design for post-Fordist science. Isis, 99(4), 796-802. Recuperado de http://www.jstor.org/stable/10.1086/595773

Glaeser, E. (2011). El triunfo de las ciudades. Cómo nuestra mejor creación nos hace más ricos, más inteligentes, más ecológicos, más sanos y más felices. Madrid: Taurus.

Graham, S. (2004). Vertical geopolitics: Baghdad and after. Antipode, 36, 12-23.

http://dx.doi.org/10.1111/j.1467-8330.2004.00379.x

Graham, S. (2016). Vertical: The city from satellites to bunkers. New York: Verso.

Grubbauer, M. (2010). The high-rise office tower as a global 'type': Exploring the architectural world of Getty Images and Co. En M. Guggenheim \& O, Söderström (Eds.), Re-shaping cities. How global mobility transforms architecture and urban form (63-80). London: Routledge.

Gottdiener, M. (1994). The social production of urban space. Austin: University of Texas Press.

Gottdiener, M. \& Budd, L. (2005). Key concepts in urban studies. London: SAGE Publications.

Gottdiener, M. \& Hutchison, R. (2011). The new urban sociology (4th dition). Philadelphia: Westview Press.

Guggenheim, M. (2009). Building memory: Architecture, networks and users. Memory Studies, 2(1), 39-53. http://dx.doi.org/10.1177/1750698008097394 
Guggenheim, M. (2010). Mutable immobiles. Building conversion as a problem of quasi-technologies. En I. Farías \& T. Bender (Ed.), Urban assemblages. How Actor-Network Theory changes urban studies. London: Routledge.

Guillén, M. (2006). The Taylorized beauty of the mechanical. Scientific management and the rise of modernist architecture. Princeton: Princeton University Press.

Hall, S. (2015). City, street and citizen: The measure of the ordinary. London: Routledge.

Halbwachs, M. (1932). Chicago, experiencia étnica. En E. Martínez Gutiérrez (Ed.), Maurice Halbwachs. Estudios de morfología social de la ciudad (pp. 199-245). Madrid: Centro de Investigación Sociológicas.

Halbwachs, M. (1938). La densidad de la población. Las grandes ciudades. En E. Martínez Gutiérrez (Ed.), Maurice Halbwachs. Estudios de morfología social de la ciudad (277-288). Madrid: Centro de Investigación Sociológicas.

Halbwachs, M. (1939). La estructura morfológica de las grandes ciudades. En E. Martínez Gutiérrez (Ed.), Maurice Halbwachs. Estudios de morfología social de la ciudad (289-295). Madrid: Centro de Investigación Sociológicas.

Halbwachs, M. (2004). La memoria colectiva. Zaragoza: Prensas Universidad de Zaragoza.

Hancock, M. (2010). Dakshina Chitra: Translating the open-air Museum in Southern India. En M. Guggenheim \& O, Söderström (Ed.), Re-shaping cities. How global mobility transforms architecture and urban form (pp. 101-122). London: Routledge.

Harvey, D. (1992). Urbanismo y desigualdad social. Madrid: Siglo XXI.

Hashimoto, K. (2002). New urban sociology in Japan: The changing debates. International Journal of Urban and Region Research, 26, 726-736.

http://dx.doi.org/10.1111/1468-2427.00413

Herod, A. (2011). Scale. London: Routledge.
Hidalgo, R. y Janoschka, M. (Eds.) (2014). La ciudad neoliberal. Gentrificación y exclusión en Santiago de Chile, Buenos Aires, Ciudad de México y Madrid. Santiago de Chile: Pontificia Universidad Católica de Chile.

Himmelwright, A. L. A. (1896). High buildings. The North American Review, 163(480), pp. 580-586. Recuperado de http://www.jstor.org/stable/25118739

Ingold T. (2008) When ANT meets SPIDER: Social theory for arthropods. En C. Knappett \& L. Malafouris (Eds.), Material Agency (pp. 209-215). Boston, MA: Springer. https://doi.org/10.1007/978-0-387-74711-8 11

Jacobs, J. (1961). The death and life of great American cities. New York: Vintage Books.

Joerges, B. (1999). Do politics have artefacts? Social Studies of Science, 29(3), 411-431. http://dx.doi.org/10.1177/030631299029003004

Kärrholm, M. (2013). Building type production and everyday life: Rethinking building types through actornetwork theory and object-oriented philosophy. Environment \& Planning. Society and Space, 31(6), 1109-1124. http://dx.doi.org/10.1068/d15312

King, A. D. (2010). Notes towards a global historical sociology of building types. En M. Guggenheiml \& O. Söderström (Eds.), Re-shaping cities. How global mobility transforms architecture and urban form (pp. 24-41). London: Routledge.

Kuppinger, P. (2010). Factories, office suites, defunct and marginal spaces: Mosques in Stuttgart, Germany. En M. Guggenheim \& O. Söderström (Eds.), Re-shaping cities. How global mobility transforms architecture and urban form (pp. 63-80). London: Routledge.

Lamo de Espinosa, E. (2001). La sociología del siglo XX. Reis, Revista Española de Investigaciones Sociológica, 96, 21-49. http://dx.doi.org/10.2307/40184382

Latour, B. (1987). Science in action: How to follow scientists and engineers through society. Cambridge: Harvard University Press.

Law, J. (2002). Aircraft stories. Decentering the object in techno science. Durham: Duke University Press. 
Lebas, E. (1982). The state in British and French urban research, or the crisis of the urban question. The Sociological Review, 30, 9-30.

http://dx.doi.org/10.1111/j.1467-954X.1982.tb00071.x

Ledrut, R. (1974). El espacio social de la ciudad. Buenos Aires: Amorrortu Editores.

Lefebvre, H. (1971). De lo rural a lo urbano. Barcelona: Ediciones Península.

Lefebvre, H. (2013). La producción del espacio. Madrid: Capitán Swing.

Lussault, M. (2015). El hombre espacial. La construcción social del espacio humano. Buenos Aires: Amorrortu Editores.

Martindale, D. (1966). Prefactory remarks: The theory of the city. En M. Weber (Ed.), The city. New York: The Free Press.

Martínez López, M. (2005). Sociologías del espacio: legado teórico y productividad empírica. Reis. Revista Española de Investigaciones Sociológicas, 109, 127-154. http://dx.doi.org/10.2307/40184669

Martínez Gutiérrez, E. (2008). Presentación. Morfología social y memoria de la ciudad. En Martínez Gutiérrez. (Ed.), Maurice Halbwachs. Estudios de morfología social de la ciudad (pp. 1-35). Madrid: Centro de Investigación Sociológicas.

May, T., Perry, B., Galès, P. L., Sassen, S., \& Savage, M. (2005). The future of urban sociology. Sociology, 39(2), 343-370.

http://dx.doi.org/10.1177/0038038505050544

McGuirk, J. (2014). Radical cities: Across Latin America in search of a new architecture. New York: Verso.

McKenzie, R. (1923). The metropolitan community. New York: Russell \& Russell.

Merton, R. K., West, P. S., Jahoda, M., \& Selvin, H. S. (Eds.) (1963). Sociología de la vivienda. Buenos Aires: Ediciones Tres.

Milicevic, A. S. (2001). Radical intellectuals: What happened to the new urban sociology? International Journal of Urban and Region Research, 25, 759-783. http://dx.doi.org/10.1111/1468-2427.00343
Mohsenin, M. \& Sevtsuk, A. (2013). The impact of street properties on cognitive maps. Journal of Architecture and Urbanism, 37(4), 301-309. https://dx.doi.org/10.3846/20297955.2013.866864

Müller, A. \& Reichmann, W. (2015). Architecture, materiality and society: Connecting sociology of architecture with science and technology studies. London: Palgrave Macmillan UK.

$\mathrm{Ng}$, E. (Ed.) (2010). Designing high-density cities for social and environmental sustainability. London: Earthscan.

Park, R. E. \& Burgess, E. (1925). The city: Suggestions for Investigation of human behavior in the urban environment. Chicago: University of Chicago Press (Heritage of Sociology Series).

Perry, B. \& Harding, A. (2002). The future of urban sociology: Report of joint sessions of the British and American Sociological Associations. International Journal of Urban and Regional Research, 26, 844-853. http://dx.doi.org/10.1111/1468-2427.00423

Pickvance, C. G. (Ed.) (2007). Urban sociology. Critical essays. London: Routledge.

Salesses, P., Schechtner, K., \& Hidalgo, C. (2013). The collaborative image of the city: Mapping the inequality of urban perception. PLoS ONE, 8(7), e68400. http://dx.doi.org/10.1371/journal.pone.0068400

Sassen, S. (1991). The global city. New York, London, Tokyo. Princeton: Princeton University Press.

Sassen, S. (2005). Cities as strategic sites. The Future of Urban Sociology. Sociology, 39(2), 343-370. https://dx.doi.org/10.1177/0038038505050544

Sassen, S. (2006). Territory, authority, rights: From medieval to global assemblages. Princeton, NJ: Princeton University Press.

Savage, M. (2005). Urban sociology in the third generation. The Future of Urban Sociology. Sociology, 39(2), 343-370.

https://dx.doi.org/10.1177/0038038505050544

Scaff, L. A. (2011). Max Weber in America. New Jersey: Princeton University Press.

Sennett, R. (1990). The conscience of the eye: The design and social life of cities. New York: Alfred A. Knopf. 
Sevtsuk, A. \& Kalvo, R. (2017). Patronage of urban commercial clusters: A network-based extension of the Huff model for balancing location and size. Environment and Planning B: Urban Analytics and City Science, 1-21.

https://doi.org/10.1177/2399808317721930

Simmel, G. (2009). Roma, Florencia, Venecia. Barcelona: Gedisa Editorial.

Simmel, G. (2014). Sociología: estudios sobre las formas de socialización. Buenos Aires: Fondo de Cultura Económica.

Star, S. L. \& Griesemer, J. R. (1989). Institutional Ecology, 'Translations' and boundary objects: Amateurs and professionals in Berkeley's Museum of Vertebrate Zoology, 1907-39. Social Studies of Science, 19(3), 387420.

Sombart, W. (1958). Lujo y capitalismo. Buenos Aires: Guillermo Dávalos Editor.

Sombart, W. (1988). La gran ciudad. En M. Bassols, R. Donoso, A. Massolo y A. Méndez (Eds.), Antología de sociología urbana (76-88). México: Universidad Nacional Autónoma de México.

Sullivan, L. H. (1896). The tall office building artistically considered. Lippincott's Magazine, 57, 403-409.

Thrasher, F. M. (2013). The gang: A study of 1,313 Gangs in Chicago. Chicago: The University of Chicago Press.

Tönnies, F. (1947). Comunidad y sociedad. Buenos Aires: Losada.

Ullán de la Rosa, F. J. (2014). Sociología urbana: de Marx y Engels a las escuelas posmodernas. Madrid: Centro de Investigaciones Sociológicas.

Venkatesh, S. (1997). The social organization of street gang activity in an urban ghetto. American Journal of Sociology, 103(1), 82-111.

https://doi.org/10.1086/231172

Venkatesh, S. (2008). Gang leader for a day. A rogue sociologist takes to the streets. New York: Penguin Books.

Venkatesh, S. (2013). Underground markets as fields in transition: Sex work in New York City. Social Forum, 28, 682-699. http://dx.doi.org/10.1111/socf.12053
Vergara Vidal, J. (2017). Verticalización. La edificación en altura en la Región Metropolitana de Santiago (19902014). Revista INVI, 32(90), 9-49. https://dx.doi.org/10.4067/s0718-83582017000200009

Weber, M. (2005). Economía y sociedad. México: Fondo de Cultura Económica.

Winner, L. (1980). Do artifacts have politics? Daedalus, 109(1), 121-136. Recuperado de http://www.jstor.org/stable/20024652

Wirth, L. (1925). A bibliography of the urban community. En R. E. Park \& E. Burgess (Eds.), The city: Suggestions for investigation of human behavior in the urban environment (161-228). Chicago: University of Chicago Press.

Wirth, L. (1998). The ghetto. New Brunswick: Transaction Publishers.

Yau, Y. (2011). Collectivism and activism in housing management in Hong Kong. Habitat International, 35 327- 334. https://doi.org/10.1016/j.habitatint.2010.11.006

Yau, Y. (2012). Normas, sentido de comunidad y 19 colectivismo comunal en un contexto de edificios en altura. Revista INVI, 76(27), 17-72. http://dx.doi.org/10.4067/S0718-83582012000300002

Yaneva, A. (2005). Scaling up and down: Extraction trials in architectural design. Social Studies of Science, 35(6), 867-894. https://doi.org/10.1177/0306312705053053

Yaneva, A. (2008). How buildings 'surprise': The renovation of the Alte Aula in Vienna. Science Studies, 21(1), 8-28. Recuperado de https://sciencetechnologystudies.journal.fi/article/view /55231

Yaneva, A. (2017). Five ways to make architecture political. An introduction to the politics of design practice. London: Bloomsbury Publishing.

Zorbaugh, H. W. (1926). The dweller in furnished rooms: An urban type. En E. Burgess (Ed.), The urban community. Selected papers from the proceedings of the American Sociological Society, 1925 (98-105). Chicago: The University of Chicago Press. 
Zorbaugh, H. W. (1976). The gold coast and the slum: Sociological study of Chicago's Near North Side. Chicago: University of Chicago Press.

Zukin, S. (1980). A Decade of the new urban sociology. Theory and Society, 9(4), 575-601. Recuperado de http://www.jstor.org/stable/656928
Zukin, S. (2011). Is there an urban sociology? Questions on a field and a vision. Sociológica, 3. http://dx.doi.org/10.2383/36415 